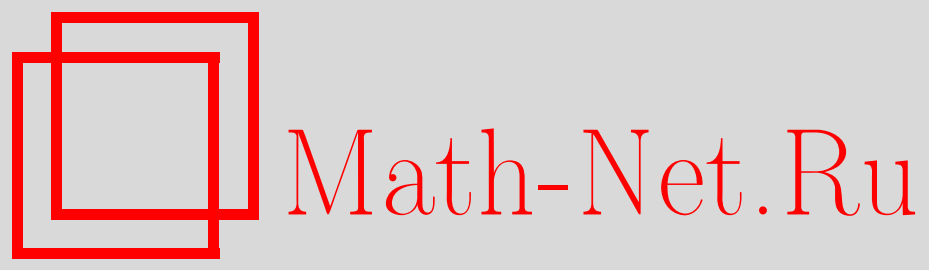

В. М. Мануйлов, Инвариант пары почти коммутирующих неограниченных операторов, Функи. анализ и его прил., 1998, том 32, выпуск 4, 8891

DOI: https://doi.org/10.4213/faa388

Использование Общероссийского математического портала MathNet.Ru подразумевает, что вы прочитали и согласны с пользовательским соглашением

http://www . mathnet.ru/rus/agreement

Параметры загрузки:

IP : 54.81 .137 .203

26 апреля 2023 г., $17: 20: 43$

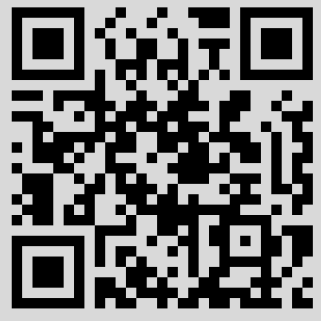




\title{
Инвариант пары почти коммутирующих неограниченных операторов ${ }^{\star}$
}

\author{
(C) 1998. В. М. МАнУйлов
}

Для широкого класса пар $(A, B)$ неограниченных симметричных операторов мы определяем $K$-теорный целочисленный инвариант $\omega(A, B)$, равный нулю для пар коммутирующих операторов, равный единице для пар $(A, B)$ со скалярным коммутатором, и обладающий свойством непрерывности. Подобные инварианты в случае ограниченных операторов были определены в работах $[1,2]$ для матриц, реализующих «почти коммутативные» тор и сферу.

1. Начнем с описания гомотопических инвариантов пар проекторов гильбертова пространства $H$ (все проекторы в настоящей заметке предполагаются самосопряженными). Обозначим через $E(A,[a, b])$ спектральный проектор оператора $A=A^{*}$, соответствующий отрезку $[a, b]$. При построении инвариантов используется следующий элементарный факт из теории возмущений.

Лемма 1 [3, с. 273]. 1) Если точка $a \in \mathbb{R}$ регулярна для оператора $A$ и $\operatorname{dist}(a, \operatorname{Sp} A)=h$, то для любого $B=B^{*}$, такого, что $\|A-B\|<h$, эта точка также регулярна.

2) Если точки $a, b$ регулярны для $A$ и удалень от $\operatorname{Sp} A$ не менее, чем на $h$, то для любого самосопряженного оператора $B$, удовлетворяющего условию $\|A-B\|<h / 2$, выполнено неравенство

$$
\|E(A,[a, b])-E(B,[a, b])\| \leqslant \frac{b-a}{h^{2}}\|A-B\| .
$$

СлЕДСТВИЕ 2. Если $P, S-$ проекторы $u\|[P, S]\|<r, r<1 / 4$, mo спектры операторов $S P S u(I-S) P(I-S)$ лежат в $[0,2 r] \cup[1-2 r, 1]$.

ДокаЗАТЕльство. Применим первое утверждение леммы 1 к операторам $A=P, B=S P S+(I-S) P(I-S)$. Заметим, что $A-B=[S,[S, P]]$; поэтому $\|A-B\|<2 r$, откуда вытекает требуемое.

Приступим к описанию инвариантов.

а) Пусть $P, S$ - проекторы, причем $\operatorname{dim} S(H)<\infty$ и $\|[S, P]\|<1 / 4$. Согласно следствию 2 , точка $1 / 2$ регулярна для $S P S$. Положив

$$
f(P, S):=\operatorname{dim} E(S P S,[-1 / 2,1 / 2])(H),
$$

получим гомотопический инвариант пар $(P, S)$ со свойством $\|[S, P]\|<1 / 4$.

Лемма 3. Пусть $P, S, P_{i}, S_{i}, i=1,2$, - проекторь.

1) Если $\left\|\left[P_{i}, S\right]\right\|<1 / 20 u\left\|S\left(P_{1}-P_{2}\right)\right\|<1 / 20, \operatorname{mo} f\left(P_{1}, S\right)=f\left(P_{2}, S\right)$.

2) Если $S_{1} S_{2}=0$ u $\left\|\left[S_{i}, P\right]\right\|<1 / 20$, mo $f\left(P, S_{1}+S_{2}\right)=f\left(P, S_{1}\right)+$ $f\left(P, S_{2}\right)$.

* Работа частично поддержана РФФИ (грант № 96-01-00276) и INTAS (грант No. 961099). 
ДокАЗАТЕльство. 1) Применим следствие 2 и второе утверждение леммы 1 к операторам $A=S P_{1} S, B=S P_{2} S$; при этом $[a, b]=[-1 / 2,1 / 2]$, $h=2 / 5$. Тогда

$$
\left\|E\left(S P_{1} S,[-1 / 2,1 / 2]\right)-E\left(S P_{2} S,[-1 / 2,1 / 2]\right)\right\|<1 ;
$$

следовательно, размерности указанных спектральных проекторов совпадаЮТ.

2) Действуем аналогично с операторами $A=S_{1} P S_{1}+S_{2} P S_{2}, B=$ $\left(S_{1}+S_{2}\right) P\left(S_{1}+S_{2}\right)$. При этом $\|A-B\|=\left\|\left[\left[P, S_{1}\right], S_{2}\right]\right\|<1 / 10$ с прежними $[a, b]$ и $h$.

б) Пусть $P, Q$ - проекторы, а $K=P-Q$ - компактный оператор. Возьмем проектор $S$, такой, что

$$
\operatorname{dim} S(H)<\infty, \quad\|[P, S]\|<\frac{1}{60}, \quad\|[Q, S]\|<\frac{1}{60}, \quad\|(I-S) K\|<\frac{1}{60},
$$

и положим $\alpha(P, Q):=f(P, S)-f(Q, S)$.

Докажем, что эта величина не зависит от выбора $S$. Для этого возьмем проекторы $S_{1}, S_{2}$, удовлетворяющие (1). Существует такой проектор $S$, что $S_{i} \leqslant S, i=1,2,[P, S]=0,\|(I-S) K\|<1 / 60$. Тогда $\|[Q, S]\|=\|[K, S]\|=$ $\|[K, I-S]\|<1 / 30$. В силу леммы $3 f(P, S)=f\left(P, S_{i}\right)+f\left(P, S-S_{i}\right)$, $f(Q, S)=f\left(Q, S_{i}\right)+f\left(Q, S-S_{i}\right)$ и $f\left(P, S-S_{i}\right)=f\left(Q, S-S_{i}\right), i=1,2$. Отсюда $f(P, S)-f(Q, S)=f\left(P, S_{i}\right)-f\left(Q, S_{i}\right)$, что доказывает корректность определения $\alpha(P, Q)$. Мы получили гомотопический инвариант, определенный для пар проекторов $P, Q$ с компактной разностью.

2. Пусть $A, B$ - симметричные операторы, определенные на области $\mathscr{D}_{0} \subset H$, такой, что $\overline{\mathscr{D}_{0}}=H$. Положим $C=\overline{A+i B}$. Определим инвариант $\omega(A, B)$ при следующих предположениях:

(i) оператор $\left(I+C^{*} C\right)^{-1}$ компактен,

(ii) замыкание образа оператора $C$ имеет конечную коразмерность в $H$.

Положим $\Delta_{0}=I+C^{*} C, \Delta_{1}=I+C C^{*}$. В гильбертовом пространстве $H \oplus H$ введем операторы

$$
P=P(C)=\left(\begin{array}{cc}
\Delta_{0}^{-1} & \left(C \Delta_{0}^{-1}\right)^{*} \\
C \Delta_{0}^{-1} & I-\Delta_{1}^{-1}
\end{array}\right), \quad Q=\left(\begin{array}{cc}
0 & 0 \\
0 & I
\end{array}\right)
$$

(оператор $P$ определен по аналогии с проектором, задающим образующую Ботта в группе $\left.\widetilde{K}^{0}\left(\mathbb{R}^{2}\right)\right)$.

Лемма 4. Операторы $P$ и $Q$ являются проекторами, а оператор $P-Q$ компактен.

ДокАЗАТЕльство. Равенство $P^{2}=P$ проверяется прямым вычислением. Для доказательства компактности оператора $P-Q$ воспользуемся полярным разложением (см. [4]) $C=V K$, где $K=K^{*} \geqslant 0$, а $V$ - частичная изометрия с областью изометричности $\overline{\operatorname{Im} K}$. Пусть $P_{i j}, i, j=1,2$, - матричные элементы оператора $P, P_{11}=\Delta_{0}^{-1}=\left(I+K^{2}\right)^{-1}, P_{21}=C \Delta_{0}^{-1}=V K\left(I+K^{2}\right)^{-1}=$ $V g\left(P_{11}\right)$, где $g(t)=\left(t-t^{2}\right)^{1 / 2}$. По условию оператор $P_{11}$ компактен. Но тогда компактен и оператор $g\left(P_{11}\right)$, откуда следует компактность операторов $P_{21}$ 
и $P_{12}=P_{21}^{*}$. Наконец, компактность оператора $P_{22}-I=-\Delta_{1}^{-1}$ вытекает из равенства $\Delta_{1}^{-1}=P_{H \ominus H_{1}}+V\left(I+K^{2}\right)^{-1} V^{*}$, где $H_{1}=V(H)=\overline{\operatorname{Im} C}$.

Положим $\omega(A, B):=\alpha(P, Q)$.

3. Пусть $A, B$ - симметрические операторы с областью определения $\mathscr{D}_{0} \subset H, \overline{\mathscr{D}_{0}}=H, A \mathscr{D}_{0} \subset \mathscr{D}_{0}, B \mathscr{D}_{0} \subset \mathscr{D}_{0}$ и $\overline{\left(I+C^{*} C\right) \mathscr{D}_{0}}=H$, где $C=$ $\overline{A+i B}$. Пусть даны также симметричные операторы $K_{i}: \mathscr{D}_{0} \rightarrow \mathscr{D}_{0}, i=1,2$, и оператор $K_{1}+i K_{2} \varepsilon$-подчинен оператору $C$ в следуюшем смысле:

$$
\|K \xi\| \leqslant \varepsilon(\|\xi\|+\|C \xi\|), \quad\left\|K^{*} \xi\right\| \leqslant \varepsilon(\|\xi\|+\|C \xi\|), \quad \xi \in \mathscr{D}_{0} .
$$

ПРЕДЛОЖЕНИЕ 5. Если $\varepsilon$ в (2) достаточно мало, т.е. меньше некоторого иисла $\varepsilon_{0}$, зависящего от $C$, то $\omega\left(A+K_{1}, B+K_{2}\right)=\omega(A, B)$.

ДокАЗАТЕльство. Положим $T=C+K$ и построим для операторов $T$ и $C$ проекторы $P(T)$ и $P(C)$. Надо показать, что $\|P(T)-P(C)\| \rightarrow 0$ при $\varepsilon \rightarrow 0$. Для этого достаточно доказать, что близки левые нижние элементы указанных проекторов. Легко проверить, что на плотном подпространстве $\left(I+C^{*} C\right) \mathscr{D}_{0} \subset H$ имеет место тождество

$$
\begin{aligned}
& T\left(I+T^{*} T\right)^{-1}-C\left(I+C^{*} C\right)^{-1} \\
& \quad=K\left(I+C^{*} C\right)^{-1}-T\left(I+T^{*} T\right)^{-1}\left(K^{*} C+T^{*} K\right)\left(I+C^{*} C\right)^{-1},
\end{aligned}
$$

и, так как $\left\|T\left(I+T^{*} T\right)^{-1}\right\| \leqslant 1 / 2,\left\|T\left(I+T^{*} T\right)^{-1} T^{*}\right\| \leqslant 1$ (это следует из полярного разложения), достаточно показать, что $\left\|K^{*} C\left(I+C^{*} C\right)^{-1}\right\| \rightarrow 0$, $\left\|K\left(I+C^{*} C\right)^{-1}\right\| \rightarrow 0$ при $\varepsilon \rightarrow 0$. Но это следует из того, что оператор $K$ подчинен оператору $C$.

4. Вычислим инвариант $\omega(A, B)$ в двух важных случаях.

ПРЕДЛОЖЕНИЕ 6. Если операторы $A$ и $B$ коммутируют (т.е. имеют коммутируюшие резольвенть $)$, то $\omega(A, B)=0$.

ДокаЗАтЕльство. Существует такой ортонормированный базис $\left\{e_{k}\right\}_{k \in \mathbb{N}}$ $\subset H$, что $C e_{k}=\lambda_{k} e_{k}, \lambda_{k} \rightarrow \infty$ при $k \rightarrow \infty$. Имеем разложение $H \oplus H=$ $\bigoplus_{j, k=1}^{\infty} H_{j k}$, где $H_{j k}$ - двумерное пространство с базисом $\left(e_{j}, 0\right),\left(0, e_{k}\right)$. Проекторы $P=P(C)$ и $Q$ оставляют каждое $H_{j k}$ инвариантным, и их действие в $H_{j k}$ задается в указанном базисе матрицами

$$
\left(\begin{array}{cc}
\left(1+\left|\lambda_{k}\right|^{2}\right)^{-1} & \bar{\lambda}_{k}\left(1+\left|\lambda_{k}\right|^{2}\right)^{-1} \\
\lambda_{k}\left(1+\left|\lambda_{k}\right|^{2}\right)^{-1} & \left|\lambda_{k}\right|^{2}\left(1+\left|\lambda_{k}\right|^{2}\right)^{-1}
\end{array}\right) \quad \text { и } \quad\left(\begin{array}{cc}
0 & 0 \\
0 & 1
\end{array}\right) .
$$

Рассмотрим проектор $S=S_{n}$ на подпространство $\bigoplus_{j, k=1}^{n} H$. Тогда $[S, P]=$ $[S, Q]=0$ и $\|S(P-Q)\| \rightarrow 0$ при $n \rightarrow \infty$. Ясно, что $f(P, S)=f(Q, S)=n^{2}$, откуда $\alpha(P, Q)=0$.

ПреДЛОЖЕНИЕ 7. Пусть $A=x, B=-i d / d x$ с естественной областью определения в $L^{2}(\mathbb{R})=H$. Тогда $\omega(A, B)=1$.

ДоКАЗАТЕЛЬСТво. Для вычислений воспользуемся базисом собственных векторов $\left\{\varphi_{n}\right\} \in H, n=0,1, \ldots$, оператора $\Delta_{0}, \Delta_{0} \varphi_{n}=(2 n+1) \varphi_{n}$. Пусть $R_{n}$ - проектор в $H$ на линейную оболочку векторов $\varphi_{0}, \ldots, \varphi_{n-1}$. Тогда в качестве проектора $S$ в $H \oplus H$ можно взять $R_{n} \oplus R_{n}$ при достаточно 
большом $n$. Поскольку $S$ коммутирует с проектором $Q$ на второе слагаемое в $H \oplus H$, условия (1) выполнены при достаточно больших $n$, так как $\|[P, S]\|=\sqrt{2(n+1)} /(2 n+3)$. Поскольку $\omega(A, B)=f(P, S)-f(Q, S)$, а $f(Q, S)=n$, остается вычислить $f(P, S)$. Для этого рассмотрим оператор $S P S$. В указанном базисе этот оператор является блочно-диагональным, $S P S=P_{0} \oplus P_{1} \oplus \cdots \oplus P_{n-1} \oplus P_{n}$, где блоки $P_{0}$ и $P_{n}$ одномерны, $P_{0}=1$, $P_{n}=(2 n+2) /(2 n+3)$ (таким образом, $P_{n} \rightarrow 1$ при $\left.n \rightarrow \infty\right)$, а блоки $P_{1}, \ldots, P_{n-1}$ двумерны и являются проекторами на одномерные пространства,

$$
P_{k}=\left(\begin{array}{cc}
\frac{2 k}{2 k+1} & \frac{\sqrt{2 k}}{2 k+1} \\
\frac{\sqrt{2 k}}{2 k+1} & \frac{1}{2 k+1}
\end{array}\right), \quad k=1, \ldots, n-1 .
$$

Следовательно, каждый блок в $S P S$ дает единичный вклад в $f(P, S)$, откуда вытекает, что $f(P, S)=n+1$.

Автор глубоко благодарен Р. С. Исмагилову за ряд полезных замечаний, существенно улучшивших настоящую заметку.

\section{ЛитеРАТУРА}

1. Choi M.-D. Proc. Amer. Math. Soc., 102, 528-533 (1988). 2. Exel R., Loring T. A. J. Funct. Anal., 95. 364-376 (1991). 3. Като T. Теория возмущений линейных операторов. Мир, М., 1972. 4. Рид М., Саймон Б. Методы современной математической физики. Т. 1. Функциональный анализ. Мир. М., 1977.

Московский государственный университет механико-математический факультет e-mail:manuilov@mech.math.msu.su
Поступило в редакцию 25 февраля 1997 г. В переработанном виде 23 марта 1998 г. 